\title{
THE DICHROMATIC NUMBER OF INFINITE FAMILIES OF CIRCULANT TOURNAMENTS
}

\author{
NAHid JaVier ANd Bernardo Llano \\ Departamento de Matemáticas \\ Universidad Autónoma Metropolitana Iztapalapa \\ San Rafael Atlixco 186, Colonia Vicentina \\ 09340, México, D.F., Mexico \\ e-mail: \{nahid,llano\}@xanum.uam.mx
}

\begin{abstract}
The dichromatic number $d c(D)$ of a digraph $D$ is defined to be the minimum number of colors such that the vertices of $D$ can be colored in such a way that every chromatic class induces an acyclic subdigraph in $D$. The cyclic circulant tournament is denoted by $T=\vec{C}_{2 n+1}(1,2, \ldots, n)$, where $V(T)=\mathbb{Z}_{2 n+1}$ and for every jump $j \in\{1,2, \ldots, n\}$ there exist the arcs $(a, a+j)$ for every $a \in \mathbb{Z}_{2 n+1}$. Consider the circulant tournament $\vec{C}_{2 n+1}\langle k\rangle$ obtained from the cyclic tournament by reversing one of its jumps, that is, $\vec{C}_{2 n+1}\langle k\rangle$ has the same arc set as $\vec{C}_{2 n+1}(1,2, \ldots, n)$ except for $j=k$ in which case, the arcs are $(a, a-k)$ for every $a \in \mathbb{Z}_{2 n+1}$. In this paper, we prove that $d c\left(\vec{C}_{2 n+1}\langle k\rangle\right) \in\{2,3,4\}$ for every $k \in\{1,2, \ldots, n\}$. Moreover, we classify which circulant tournaments $\vec{C}_{2 n+1}\langle k\rangle$ are vertex-critical $r$-dichromatic for every $k \in\{1,2, \ldots, n\}$ and $r \in\{2,3,4\}$. Some previous results by Neumann-Lara are generalized.
\end{abstract}

Keywords: tournament, dichromatic number, vertex-critical $r$-dichromatic tournament.

2010 Mathematics Subject Classification: 05C20, 05C38.

\section{REFERENCES}

[1] G. Araujo-Pardo and M. Olsen, A conjecture of Neumann-Lara on infinite families of r-dichromatic circulant tournaments, Discrete Math. 310 (2010) 489-492. doi:10.1016/j.disc.2009.03.028

[2] J. Bang-Jensen and G. Gutin, Digraphs. Theory, Algorithms and Applications, Second Edition (Springer Monographs in Mathematics, Springer-Verlag London, London, 2009). 
[3] P. Erdős, Problems and results in number theory and graph theory, Proceedings of the Ninth Manitoba Conference on Numerical Mathematics and Computing (Univ. Manitoba, Winnipeg, Man., 1979), Congr. Numer. XXVII (1979) 3-21.

[4] P. Erdős, J. Gimbel and D. Kratsch, Some extremal results in cochromatic and dichromatic theory, J. Graph Theory 15 (1991) 579-585. doi:10.1002/jgt.3190150604

[5] A. Harutyunyan, Brooks-type results for coloring of digraphs, $\mathrm{PhD}$ thesis supervised by B. Mohar (Simon Fraser University, 2011). http://www.math.univ-toulouse.fr/ aharutyu/thes-short.pdf

[6] H. Jacob and H. Meyniel, Extensions of Turan's and Brooks theorem and new notions of stability and colouring in digraphs, Ann. Discrete Math. 17 (1983) 365-370.

[7] B. Llano and M. Olsen, On a conjecture of Victor Neumann-Lara, Electron. Notes Discrete Math. 30 (2008) 207-212. doi:10.1016/j.endm.2008.01.036

[8] B. McKay, Combinatorial Data, published online. http://cs.anu.edu.au/ bdm/data

[9] V. Neumann-Lara, The dichromatic number of a digraph, J. Combin. Theory, Ser. B 33 (1982) 265-270. doi:10.1016/0095-8956(82)90046-6

[10] V. Neumann-Lara, The 3 and 4-dichromatic tournaments of minimum order, Discrete Math. 135 (1994) 233-243. doi:10.1016/0012-365X(93)E0113-I

[11] V. Neumann-Lara, Vertex critical 4-dichromatric circulant tournaments, Discrete Math. 170 (1997) 289-291. doi:10.1016/S0012-365X(96)00128-8

[12] V. Neumann-Lara, Dichromatic number, circulant tournaments and Zykov sums of digraphs, Discuss. Math. Graph Theory 20 (2000) 197-207. doi:10.7151/dmgt.1119

[13] V. Neumann-Lara and J. Urrutia, Vertex critical r-dichromatric tournaments, Discrete Math. 49 (1984) 83-87. doi:10.1016/0012-365X(84)90154-7

Received 30 June 2016

Revised 7 April 2016

Accepted 7 April 2016 